\title{
An Extracellular Matrix-Liposome Composite, a Novel Extracellular Matrix Delivery System for Accelerated Tissue Regeneration
}

\author{
Keel Yong Lee, Huong Thanh Nguyen, Agustina Setiawati, So-Jung Nam, Minyoung Kim, \\ II-Gyu Ko, Won Hee Jung, Kevin K. Parker, * Chang-Ju Kim,* and Kwanwoo Shin*
}

The unfolded states of fibronectin (FN) subsequently induce the formation of an extracellular matrix (ECM) fibrillar network, which is necessary to generate new substitutive tissues. Here, the authors demonstrate that negatively charged small unilamellar vesicles (SUVs) qualify as candidates for FN delivery due to their remarkable effects on the autonomous binding and unfolding of FN, which leads to increased tissue regeneration. In vitro experiments revealed that the FN-SUV complex remarkably increased the attachment, differentiation, and migration of fibroblasts. The potential utilization of this complex in vivo to treat inflammatory colon diseases is also described based on results obtained for ameliorated conditions in rats with ulcerative colitis (UC) that had been treated with the FN-SUV complex. Their findings provide a new ECM-delivery platform for ECM-based therapeutic applications and suggest that properly designed SUVs may be an unprecedented FN-delivery system that is highly effective in treating UC and inflammatory bowel diseases. favor interactions with various cell types, growth factors, cytokines, and other ECM proteins, such as collagen, and heparin. ${ }^{[2-4]}$ Soluble FN is a folded dimeric protein whose subunits are composed of three types of repeating modules, I, II, and III with alternatively spliced $\mathrm{V}$ domains, which account for various isoforms of plasma FN. ${ }^{[5,6]}$ Among the three modules, FN type III (FNIII) is the largest and most common repeat. ${ }^{[2,4]}$ Once in contact with the cell surface, FNIII domains will be recognized by integrin proteins on the cell membrane and subsequently undergo an unfolding process caused by cell-traction forces, which induces FN fibrillogenesis..$^{[3,7,8]} \mathrm{Un}$ folding is, therefore, essential in FN functionalization, which is the target of $\mathrm{FN}$ based material fabrication. ${ }^{[1,9]}$ Although the cell binding sites within the FNIII module are exposed on the surface, the active domains, which are known to be phosphorylation sites, are usually hydrophobic. These domains are buried within FNIII modules in the folded state due to hydrophobic interactions and are maintained by intramolecular ionic bonds. ${ }^{[10,11]}$ Therefore, unfolding FN is crucial not only for enhancing the elasticity of fibronectin fibrils but also for exposing the buried sites that are known to display enzymatic activities and to regulate many cellular activities. ${ }^{[12]}$
K. Y. Lee, H. T. Nguyen, A. Setiawati, S.-J. Nam, M. Kim, K. Shin

Department of Chemistry and Institute of Biological Interfaces

Sogang University

Seoul 04107, Republic of Korea

E-mail: kwshin@sogang.ac.kr

K. Y. Lee, K. K. Parker, K. Shin

Disease Biophysics Group

John A. Paulson School of Engineering and Applied Sciences

Harvard University

Cambridge 02138, USA

E-mail: kkparker@seas.harvard.edu

The ORCID identification number(s) for the author(s) of this article can be found under https://doi.org/10.1002/adhm.202101599

DOI: 10.1002/adhm.202101599

\author{
A. Setiawati \\ Department of Life Science \\ Sogang University \\ Seoul 02447, Republic of Korea \\ A. Setiawati \\ Faculty of Pharmacy \\ Sanata Dharma University \\ Yogyakarta 55284, Indonesia \\ I.-G. Ko, C.-J. Kim \\ Department of Physiology \\ College of Medicine \\ Kyung Hee University \\ Seoul 02447, Republic of Korea \\ E-mail: changju@khu.ac.kr \\ W. H. Jung \\ Department of Systems Biotechnology \\ Chung-Ang University \\ Anseong 17546, Republic of Korea
}


The use of soluble, compact FN has been successful in improving wound-healing treatments after being unfolded by various mechanisms. ${ }^{[13,14]}$ Some examples of wounds considered thus far are diabetes- and radiation-induced cutaneous wounds in rats or mice. ${ }^{[15,16]}$ Corneal epithelial wounds have also been reported to heal more quickly after having been treated using eye drops containing a soluble FN and hyaluronan combination. ${ }^{[17]}$ Various efforts have also been made to develop large-scale fibronectin networks which might be adopted to facilitate the wound healing process ${ }^{[18-20]}$ Nevertheless, recent reports have revealed that supplying cells with unfolded FN, compared to compact FN, improves the wound-healing efficiency more remarkably. For example, Le et al. reported an enhanced formation of FN fibrils after denaturation of FN by using urea, leading to improved platelet adhesion. ${ }^{[21]}$ In 2018, Christophe et al. adopted rotary jet spinning to produce unfolded FN fibers and confirmed that its use effectively enhanced wound healing. ${ }^{[22]}$ Most recently, Neale et al. demonstrated that the aligned network of unfolded FN was critical for directing fibroblast orientation and migration. ${ }^{[23]}$ These studies suggest that for higher wound-healing efficiency, FN used in tissue regeneration should undergo certain procedures to induce changes in its conformation. Some methods for doing so include physical binding on different substrates, modifying surfaces with chemical groups, or using gold nanoparticles. ${ }^{[24-28]} \mathrm{Al}-$ though unfolded FN has been shown to perform better than compact FN, previously reported methods for unfolding FN have had limited clinical application due to the complexity of the processes required. Urea-induced FN unfolding has also been used, but it has not been widely used in in vivo experiments due to its potential cytotoxicity. [29-31]

In this study, to tackle the above issues, we demonstrate a potential FN-unfolding platform that uses negatively charged, small unilamellar vesicles (SUVs) for FN delivery. These SUVs are simply produced by using a unique composition of lipids so that the surface of the SUV not only enhances direct binding but also induces the unfolding of FN. The effects of FN delivered by our SUV system were investigated using a variety of cell-based assays to evaluate all aspects of cellular activities, including growth, differentiation, and migration. An in vivo wound-healing model of ulcerative colitis (UC) was adopted to evaluate the sufficiency of the unfolded FN that was delivered. Collectively, this FN-SUV system provides a platform that can be easily integrated into existing drug delivery systems due to its high biocompatibility, homogeneous production via spontaneous FN binding, and, provisionally, ease of formulation with a variety of drugs.

\section{Results}

\subsection{FN Stretched after Binding on Negatively Charged Liposomes}

For critical cellular functions to be accessed, compact FN must be unfolded to expose buried domains. ${ }^{[32]}$ Hence, our first effort was to determine the composition of lipids that not only enhanced the binding efficiency of FN but also induced conformational changes of FN prior to its delivery to cells. The optimized lipid composition of SUVs was found to be zwitterionic:net negative:cholesterol at a ratio of 3:2:1. An appropriate ratio of 1,2dioleoyl-sn-glycero-3-phospho-L-serine (DOPS) resulted in a net negative charge on the surface of the SUV (Figure 1a). An analogous giant unilamellar vesicle (GUV) system was developed with the same lipid composition but a hundredfold increase in size (20-30 $\mu \mathrm{m}$ on average) (Figure 1b,c). The analogous GUVs allowed us to observe the binding directly by using confocal imaging. FN tended to form insoluble aggregates when it was incubated with neutral vesicles (Figure $1 \mathrm{~b}$ and Figure S1a, Supporting Information), but was attached evenly on the surfaces of negatively charged GUVs (Figure 1c and Figure S1b, Supporting Information). The strongly favorable and selective binding of FN to a negatively charged lipid is obvious in phase-separated GUVs with two lipid phases (Figure S2, Supporting Information). The liquid order $\left(L_{o}\right)$ phase contained zwitterionic sphingomyelin, and the liquid disorder $\left(L_{\mathrm{d}}\right)$ phase contained DOPS and other unsaturated lipids, constructing the negative portion of the membrane. Under the same experimental conditions, FN was observed only in the $L_{\mathrm{d}}$ phase (green fluorescence) and not in the $L_{\mathrm{o}}$ phase of the same GUV (Figure S2b, Supporting Information). This result clearly confirmed that a negatively charged vesicle system selectively enhanced FN binding.

Direct evaluation of the binding of FN on SUVs with an appropriate size for cell delivery was much more sophisticated because SUVs (average radius of $200 \mathrm{~nm}$ ) are remarkably smaller than GUVs (average radius of $20 \mu \mathrm{m}$ ). Images obtained from cryogenic transmission electron microscopy (cryo-TEM) showed that the SUVs incubated with FN had a membrane that was significantly thicker (thickness $d=8.3 \pm 0.6 \mathrm{~nm}$ ) than the membrane containing only lipids $(d=4.4 \pm 0.6 \mathrm{~nm}$ ) (Figure $1 \mathrm{~d}, \mathrm{e})$. The thickness of the membrane comprises the lipid membrane itself, which is $\approx 4-5 \mathrm{~nm}$, and an additional FN layer, if any, on the membrane. This result implied that FN was, indeed, bound to the SUVs, which was observed as an increase in the membrane thickness. In addition to performing single-SUV thickness measurements, we measured the size distribution of the entire SUV population by using the dynamic light scattering (DLS) method (Figure S3, Supporting Information). The results showed that the average size of the SUV population increased from 140.3 to $178.3 \mathrm{~nm}$ and that the curve shifted to the right (larger size), indicating that the size of the SUV had been increased during incubation in an FN solution.

In addition to enhancing the binding of FN on the surface, negatively charged SUVs induced conformational changes. The stretching of FN was measured by using fluorescence resonance energy transfer (FRET) with FN labeled with donor-acceptor dyes (FN-DA). Four molar (4 M) guanidinium chloride ( $\mathrm{GdnHCl})$, one of the strongest denaturants, was used as a positive control [33] (Figure S4, Supporting Information). Figure 1f shows that the $I_{\mathrm{A}} / I_{\mathrm{D}}$ ratio of FN-DA incubated with SUVs was comparable to that of FN-DA incubated in $4-\mathrm{M} \mathrm{GdnHCl}(0.38 \pm 0.06$ and $0.34 \pm$ 0.13 , respectively) and was significantly lower than that of FN-DA incubated in phosphate buffered saline (PBS), which was used for SUV hydration, or sucrose, which was used for GUV hydration $(1.18 \pm 0.2$ and $1.19 \pm 0.05$, respectively). FRET was also accomplished on GUVs coated with FN-DA; the $I_{\mathrm{A}} / I_{\mathrm{D}}$ ratio of FNDA was decreased after incubation with GUVs (from $0.92 \pm 0.11$ to $0.18 \pm 0.06$ after incubation) (Figure $1 \mathrm{~g}, \mathrm{~h}$ ). This result verified that stretching of FN had also occurred on the surfaces of GUVs. Taken together, the negatively charged SUV system is a suitable vehicle for delivering $\mathrm{FN}$ in an active, unfolded conformation. 
a
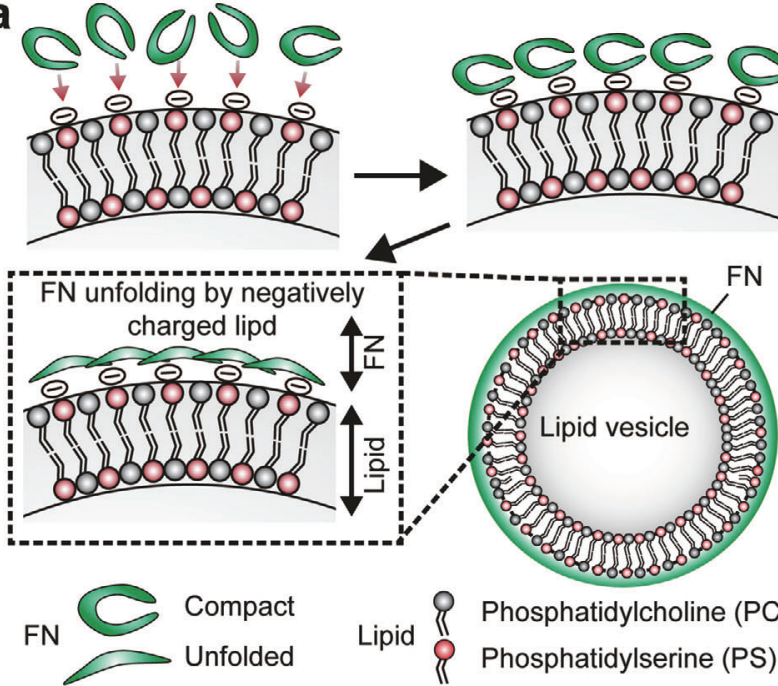
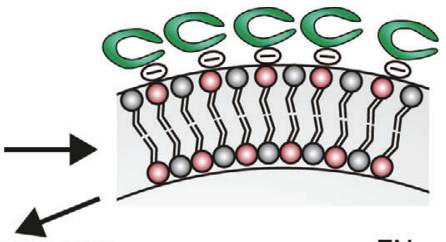

FN

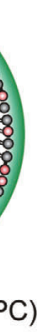

Lipid b

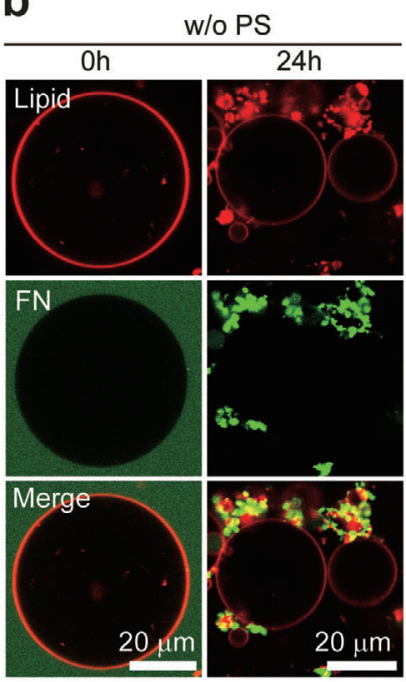

c

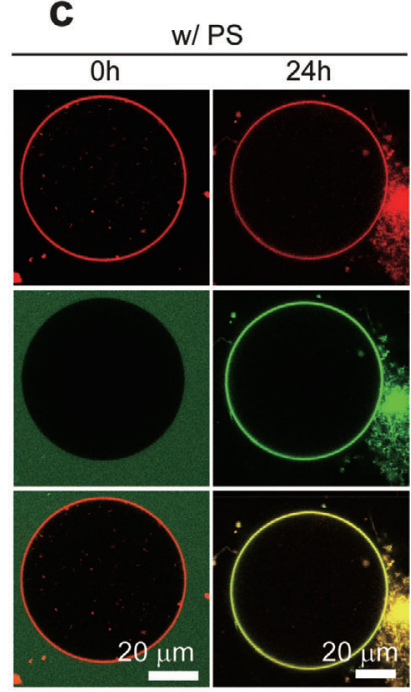

d

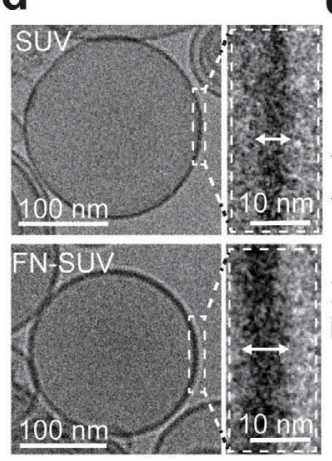

e

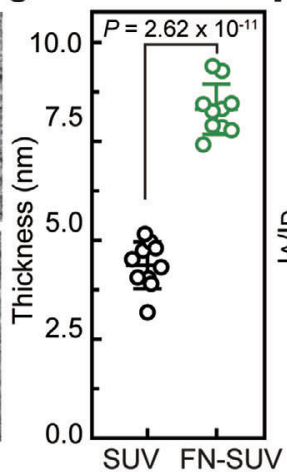

f

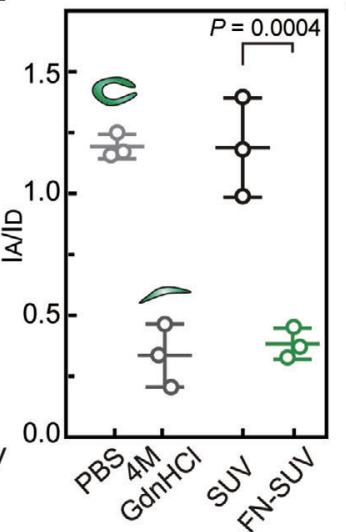

g
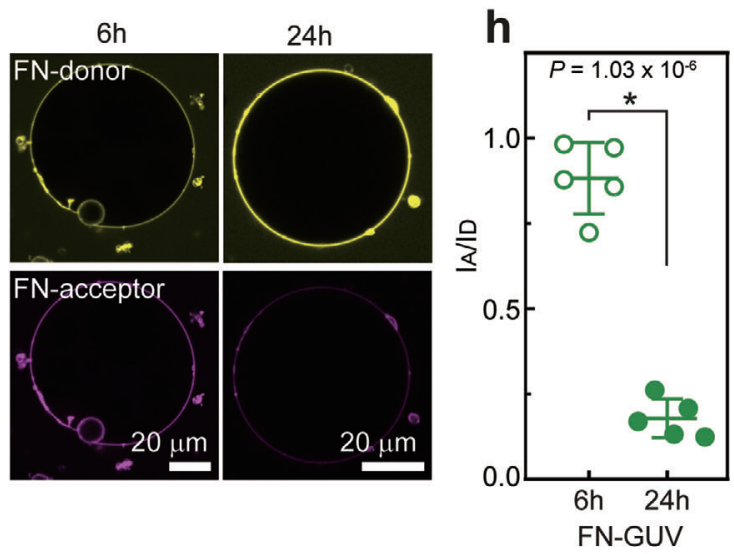

Figure 1. Fibronectin binds to negatively charged vesicles, which changes its conformation. a) Illustration showing how a negatively charged surface induces binding and stretching of fibronectin (FN). b,c) Confocal images of a GUV (red) without (b) and with (c) DOPS-containing GUVs after incubation with FN (green) for $24 \mathrm{~h}$ ( $n=5$ independent experiments per group). d) Cryo-TEM images of an SUV and magnified views of its membrane (right panels) after incubation with (lower) and without FN (upper). e) Summary of the membrane thicknesses observed in (d) ( $n=10$ per group). $f$ ) Data from FRET showing the intensity ratio $I_{\mathrm{A}} / I_{\mathrm{D}}$ observed in double-labeled $\mathrm{FN}$ diluted in PBS, $4 \mathrm{M} \mathrm{GdnHCl}$, or sucrose, or incubated with SUVs ( $n=3$ per group). IA and ID are the acceptor and the donor fluorescence intensities, respectively. $g$ ) Single-vesicle FRET using confocal imaging showing the donor and the acceptor intensities in GUVs after 6 and $24 \mathrm{~h}$ of incubation with FN. h) Summary of single-vesicle FRET from (g) showing the intensity ratios $I_{\mathrm{A}} / I_{\mathrm{D}}$ after 6 and 24 h of incubation with FN and GUVs ( $n=5$ per group). Data represent the means \pm standard deviations (SDs)

\subsection{FN-SUV Increased Cell Attachment and Proliferation at a Greater Rate than Soluble FN}

FN has been utilized in cell and tissue cultures to strengthen cell attachment, proliferation, and migration. ${ }^{[25,27,34-36]}$ We hypothesized that the conformational changes induced by binding to negatively charged SUVs would enhance the above cellular functions of FN to a greater extent than its compact form. We performed in vitro experiments using human neonatal dermal fibroblast (HDFn) cells, as they play essential roles in tissue repair. The fate of FN after having been delivered to HDFn cells was probed with either green (FN-SUV) or red (FN) fluorescent indicators. Figure 2a illustrates the formation of a FN matrix after having approached the surface of the HDFn cells. In both groups, FN fibrils were found to colocalize with their membrane receptor, integrin $\alpha 5$ (Figure 2b). However, more FN fib- rils were produced when FN was delivered by using SUVs (Figure S5, Supporting Information). Our results suggest that FNSUVs burst and expanded when they came into contact with the surface of the HDFn cells by the interaction between integrin $\alpha 5$ and FN fibrils (Figure 2c). This event was not observed on samples with aggregated FN without SUVs (data not shown), indicating that stretched FN promoted interaction with the cell surface. As we observed that SUVs unfolded FN in advance of the interaction with the cell membrane, we predicted that this would subsequently increase the adhesion of cells to the culture substrate when they are treated with FN-SUV. Figure 2d shows that trypsinized HDFn cells (green, labelled with GFP) had limited attachment to the culture dish. However, when FNSUV was added, the number of attached and active fibroblasts (seen as spindle-shaped cells with long lamellipodia) on the disk bottom was significantly increased. ${ }^{[37]}$ A quantitative compari- 

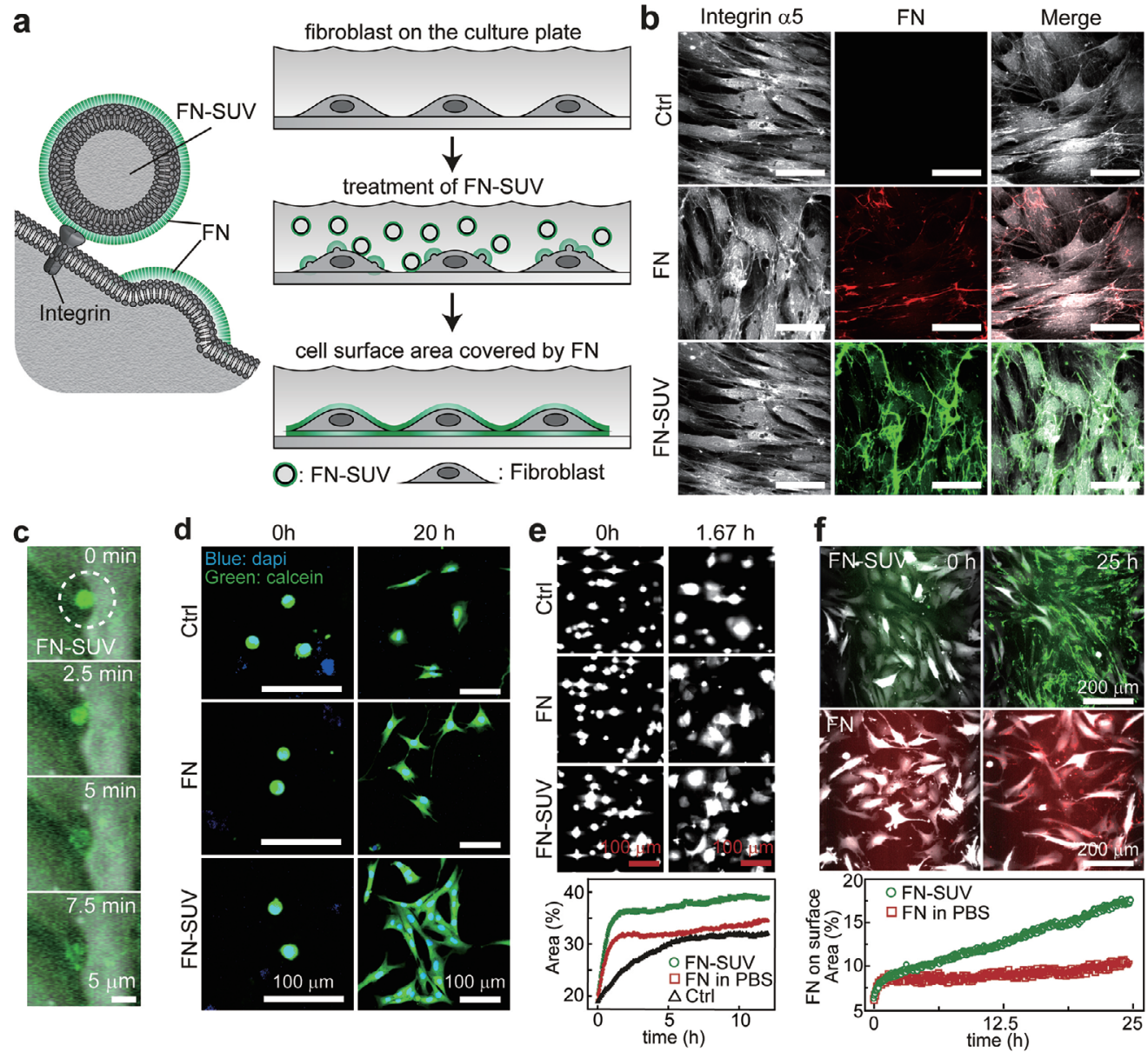

Figure 2. Delivery of FN-SUVs enhances fibrillogenesis and cell attachment. a) Schematics of FN-SUV on the HDFn surface. b) Immunostaining images illustrating binding patterns of FN fibrils (red or green) on HDFn surfaces ( $n=3$ independent experiments per group). White represents the integrin $\alpha 5$-FITC antibody. c) Images of 7.5-min time intervals showing the untangling process of FN-SUV bundles on the surfaces of HDFn cells $(n=3)$. d) Live-cell images showing changes in the morphologies of HDFn cells after $20 \mathrm{~h}$ of incubation with PBS (Ctrl), FN, or FN-SUVs ( $n=3$ independent experiments per group). e) Attachment rate of HDFn cells quantified by the area of the cells attached to the dish's surface ( $n=3$ per group). f) Live cell images showing the areas of the FN matrix formed on the surfaces of HDFn cells after incubation with FN or FN-SUVs $(n=5$ independent experiments per group).

son of cell attachment among the experimental groups was performed by calculating the total area of attached cells, as shown in Figure 2e. Moreover, the area of the FN matrix was significantly greater when FN was delivered by SUVs, implying enhanced extracellular matrix formation (Figure $2 \mathrm{f}$ and Video S4, Supporting Information). Although FN promoted cell attachment, stretched FN made the surfaces of GUVs unfavorable for bacteria, particularly Staphylococcus aureus (S. aureus), an organism that often causes opportunistic infections on skin and leads to tissue damage, as described in a previous study. ${ }^{[38]} \mathrm{S}$. aureus was incubated with different types of GUVs (Figure S6 and Videos S1-S3, Supporting Information). A large number of bacteria (white) were found on collagen-coated GUVs (COL-GUVs). In contrast to the case with the bare lipid GUVs, the bacteria were hardly present on the FN-coated GUVs, suggesting that using the stretched FN as a wound healing agent had advantages in preventing unwanted bacterial infection during the healing process.

\subsection{SUV-Induced Stretched FN Promoted Cell Migration}

When tissue is injured, released cytokines attract fibroblasts from the surrounding area..$^{[1]}$ During this time, fibroblasts are activated and migrate towards the injured area with the recruitment of ECM proteins including FN.$^{[39,40]}$ We tested whether the stretching of FN would promote the migration rate of fibroblasts, as it has been shown to enhance ECM formation. Figure 3a shows representative images cut from Videos S5-S7, Supporting Information, illustrating the movements of HDFn-GFP cells as a function of time. Single cells were tracked, and the average migration speeds (micrometer per minute) were compared among groups. As we expected, the migration speed increased when SUVs (green circles) were used for FN delivery (Figure 3a,b). This enhancement may result from the formation of a denser FN matrix, which facilitates cell adhesion and migration. The expansion of the FN matrix over time can be seen clearly in Figure S7 and Video S11, Supporting Information. 

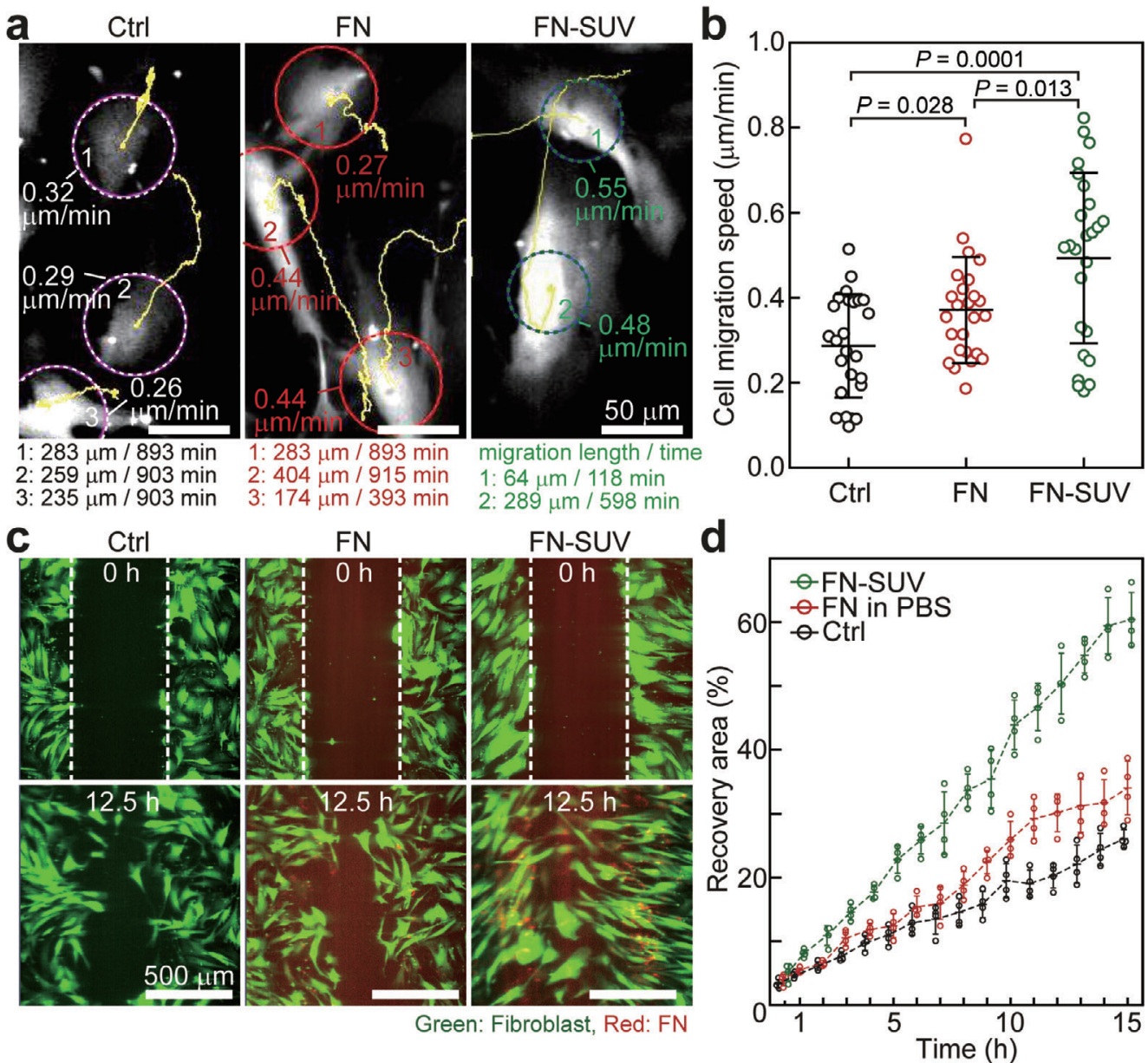

Figure 3. FN-SUV treatment accelerates the migration rate of HDFn cells. a) Representative images tracking the migration speed of single HDFn cells in the control (Ctrl), FN- and FN-SUV-treated groups. b) Summary of the single-cell migration speeds in the control, FN- and FN-SUV-treated HDFn cells ( $n=25$, from 5 independent experiments). c) in vitro scratch assays were performed in the control, FN- and FN-SUV-treated HDFn cells to compare the rates of migration. Representative images were extracted from the time series at 0 and $12.5 \mathrm{~h}$. d) Results of quantitative analyses of the recovery areas covered by migrated cells as described in (c). Data were collected from the same optical field at $1 \mathrm{~h}$ time intervals over $15 \mathrm{~h}$ ( $n=4 \mathrm{independent}$ experiments).

In vitro scratch assays were used to assess the cell migration speed to evaluate cell migration. ${ }^{[41]}$ To ensure the reproducibility of the experiments, we set up an incubation chamber in the confocal microscope system and continuously observed a specific injured area for at least $16 \mathrm{~h}$. Representative images at 0 and 12.5 $\mathrm{h}$ for each group are shown in Figure 3c; the corresponding timelapse images are shown in Videos S8-S10, Supporting Information. The results revealed that among the experimental groups, the gap closed at the highest rate in the FN-SUV-treated group, with $60.3 \pm 4.3 \%$ of the gap area being covered by HDFn cells after $15 \mathrm{~h}$ compared to only $33.5 \pm 4.2 \%$ in the FN-treated group (Figure 3d) with higher directionality and more elongated morphology (Figure S8, Supporting Information). Insignificant proliferation among all groups was observed during the first $24 \mathrm{~h}$, which showed that the cell migration assays were unbiased, with minimal contribution from cell proliferation (Figure S9, Supporting Information).

\subsection{FN-SUV Improved Wound Healing in Rats with Ulcer Colitis More Significantly than Compact FN}

The healing effects of FN in vivo were described previously by using a wide range of wound models in different species, such as rabbits, mice, and guinea pigs. ${ }^{[16,42,43]}$ Existing evidence from previous studies indicates that in inflammatory bowel-related diseases, such as ulcerative colitis (UC) and Crohn's disease, the level of FN in the plasma is decreased significantly. ${ }^{[9,44]}$ In this present study, the healing performance of FN-SUVs was further assessed in vivo using an UC model for rats. An approved protocol using 4\% acetic acid was applied to induce UC in the colon, followed by a 10-day treatment strategy with either FN or FNSUVs (Figure S10, Supporting Information). After treatment, the entire colons of the rats were removed to compare changes in morphology, wet weights, and various tissue damage markers (Figure 4a). As shown in Figure 4b, the FN-SUV-treated group 

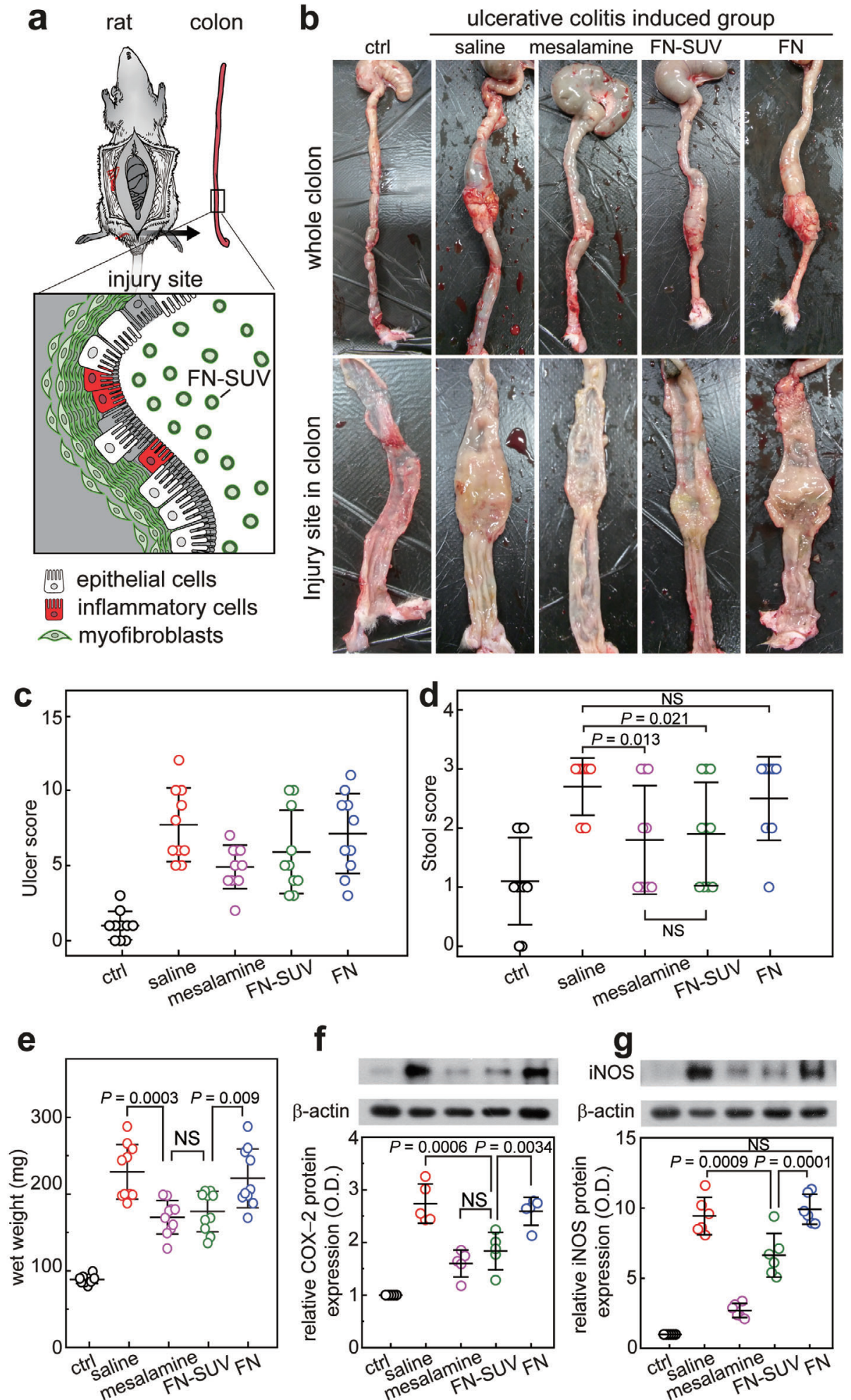

Figure 4. FN-SUV treatment improves the healing process of acid-induced ulcers in rat colons. a) Hypothesized schematic of FN-SUV treatment in the rat colon. b) Gross morphologies of the intestines removed from the rats after 10 days of treatment. In the control group, the healthy colon wall can be seen to be thin and smooth with no mucus while in the colitis-induced groups, the wall of the colon is swollen and filled with blood and mucus. Various degrees of colitis can be seen among the different treatments. c,d) Ulcer score and stool score of the control and the colitis-induced groups as described in (a) ( $n=10$ per group). e) Colon wet weight of the control and the colitis-induced groups as described in (a) ( $n=10$ per group). $p$ values were calculated using Student's $t$-tests. $f, g$ ) Expression levels of the inflammatory indicators COX-2 ( $n=5$ per group) (d) and iNOS ( $n=6$ per group) (e). $d-g)$ Error bars indicate the SDs. 
showed remarkably thinner and smoother colon walls with less mucus (less tissue damage) than the FN-treated group. Moreover, mesalamine- and FN-SUV-treated groups had decreased the ulcer scores and stool score as a result of the improvements of histological alterations, including ulceration, mucosal atrophy, submucosal edema, and inflammatory cells infiltration (Figure 4c,d and Figure S11, Supporting Information). In addition, FN-SUVtreated colons had significantly lower wet weights than those treated with FN alone, indicating that SUV-FN treatment reduced inflammation and swelling in the colons (Figure 4e).

Colon tissues after either FN or FN-SUV treatment were further analyzed semi-quantitatively for inflammatory markers, including tumor necrosis factor (TNF)- $\alpha$, interleukin (IL)-1 $\beta$, IL6 , cyclooxygenase (COX)-2, and inducible nitric oxide synthase (iNOS). However, the differences in the levels of tumor necrosis factor (TNF)- $\alpha$, IL- $1 \beta$, and IL- 6 expressions between the FN and the FN-SUV groups were insignificant (Figure S12, Supporting Information) whereas COX-2 and iNOS expressions were downregulated in rats treated with FN-SUV, suggesting that the unfolding of FN via the SUV may expose domains that communicate with cells via these two pathways (Figure 4f,g) ${ }^{[45,46]}$ The inhibitions of the COX-2 and the iNOS expressions in our in vivo model clearly showed that inflammation was effectively decreased in the experimental UC model when the FN-SUV treatment was administered. ${ }^{[47]}$

\section{Discussion}

Many attempts have been made to unfold FN prior to its reaching the cell. However, an appropriate approach to unfolding FN for tissue regeneration applications is still a challenge. The unfolding of FN requires the breakage of intramolecular bonds and hydrophobic interactions, which can be induced by using either chemicals ${ }^{[21,33]}$ or mechanical forces. ${ }^{[10,22]}$ The $\mathrm{pH}$ or salt concentration can induce the stretching of $\mathrm{FN}$, but this reversible change is hard to maintain for cell delivery. Moreover, the solution must be similar to the physiological environment. The improper removal of a denaturant will otherwise potentially result in negative effects on live cells and tissues. ${ }^{[33]}$

The adsorption and unfolding facilitated by chemical modifications raise a risk of cytotoxicity. ${ }^{[24-27]}$ On the other hand, approaches using mechanical forces to unfold FN usually exploit solid substrates and polymers with low biocompatibility. The most promising approach might be to produce nanofibers from soluble FN by using mechanical forces. ${ }^{[22,48]}$ However, the FN nanofiber production remains challenging, as the use of the fabrication technique may not always be possible in laboratories. Nonetheless, FN nanofibers are more appropriate for dressing materials and cutaneous wound treatment than for the treatment of bowel diseases. Despite major efforts to deliver ECM or FN to damaged tissues via surface-modified substrates, the fabrication of these materials remains very complex, and these substrates usually have to be removed from the body after having been applied, which is not appropriate for deep, closed wounds. ${ }^{[49,50]}$ In contrast, negatively charged SUVs are highly biocompatible, which have been used for drugs and mRNA-base vaccines. ${ }^{[51-53]}$ All the lipids (e.g., DOPC, DOPS, and DOPE) used in this research have been clinically approved and are being commercialized. ${ }^{[54]}$ Accordingly, we managed to develop a nega- tively charged SUV system that had negligible toxicity and could safely deliver the desired therapeutic materials. ${ }^{[51,55]}$ In addition, the preparation of self-assembled SUVs in an aqueous solution, followed by the autonomous binding and unfolding of FN, was much simpler and afforded higher yields than any other method.

The adsorption and unfolding of FN on liposomes were reported by Halter et al. in 2005. ${ }^{[56]}$ Both gel-phase and liquid-phase liposomes were considered for FN selective binding, but only liposomes composed of lipids that had high melting temperature $\left(T_{m}\right)$ or existed in the gel phase at room temperature, such as DPPC $\left(T_{m}=41^{\circ} \mathrm{C}\right)$ or DSPC $\left(T_{m}=55^{\circ} \mathrm{C}\right)$, were observed to bind and unfold FN effectively. ${ }^{[56]}$ Apart from gel-phase liposomes, we have sufficient experimental evidence to prove that FN is able to bind and unfold on liquid-phase liposomes composed of lipids with $T_{m}<0{ }^{\circ} \mathrm{C}$ under the condition that a negatively charged lipid (DOPS) is added. Due to the low $T_{m}$ values of the lipids used, the fabrication of negatively charged SUVs is simplified and can be performed at room temperature. Nevertheless, the in vitro and vivo effects of FN-SUV have not been characterized, although the adsorption and unfolding of FN on liposomes was reported in detailed in Halter's study. Therefore, the negatively charged SUV system has significant advantages over previous FN-based wound healing materials in terms of fabrication, cytotoxicity, and biocompatibility.

\section{Conclusions}

Collectively, we confirmed that negatively charged SUVs could effectively unfold FN bound to their surfaces. Subsequently, the SUVs enhanced the effects of FN on HDFn migration and proliferation. In vivo experiments showed that rats with UC that had been treated with FN-SUVs improved faster than rats treated with only soluble FN. The abundant amount of FN in the plasma is an advantage of using $\mathrm{FN}$ as a potential tissue regeneration material. ${ }^{[1,9,44]}$ FN also possesses an exceptional property for wound healing, as stretched FN disrupts the binding of $S$. aureus, a common cause of infections in humans. ${ }^{[38]}$

We conclude that negatively charged SUVs are ideal vehicles for functional FN delivery. The system has the capability to carry and unfold FN with high biocompatibility and safety and can be used as a platform for incorporating drugs that accelerate wound healing. Further studies are expected to discover the mechanisms underlying the efficacy of the SUV-FN delivery system.

\section{Experimental Section}

Preparation of Small Unilamellar Vesicles (SUVs) with Negatively Charged Surfaces: Multilamellar liposomes were first prepared by blending DOPC:DOPE:DOPS:cholesterol in a ratio of 2:1:2:1 to make a mixture with a total concentration of $5 \mathrm{mg}$ of lipids per milliliter in chloroform. One milliliter of the lipid blend in a black glass vial was blown dry using $\mathrm{N}_{2}$ gas for $2 \mathrm{~h}$ to completely remove the solvent. The dried lipids were then rehydrated with $1 \times$ PBS ( $\mathrm{pH} 7.4)$ by using a vortex mixer at room temperature for $60 \mathrm{~min}$. The lamellarity of the liposomes was reduced by using 5 cycles of freezing in liquid nitrogen and thawing in a water bath at $40^{\circ} \mathrm{C}$ to break down the outer bilayers. Finally, the vesicles were extruded by using a mini-extruder set (Avanti, Inc.) to pass them through a 200-nm membrane filter 11 times. The day before experiment, a fibronectin (FN) solution that had been previously diluted in deionized water was added to make an FN 
or FN-SUV solution with a final concentration of $10 \mu \mathrm{g} \mathrm{mL}^{-1}$, followed by incubation at $37^{\circ} \mathrm{C}$ overnight.

Preparation of Giant Unilamellar Vesicles (GUVs): A lipid mixture with a composition of either DOPC:DOPE:DOPS:cholesterol (2:1:1:1 molar ratio) for negatively charged GUVs or DOPC:DOPE:cholesterol (2:1:1) for neutrally charged GUVs was prepared with a concentration of $1 \mathrm{mg} \mathrm{mL}^{-1}$. Approximately $30 \mu \mathrm{L}$ of the lipid mixture was deposited on ITO glass (30Ohm sheet resistance) and quickly dried by using flowing $\mathrm{N}_{2}$ gas. The lipids were continuously dehydrated in a vacuum desiccator for $\approx 1 \mathrm{~h}$. A silicon ring with a thickness of $1 \mathrm{~mm}$ was inserted between two ITO glass slides to make space for the hydrate solution. For observations under a light microscope, the lipids were rehydrated with an inner solution containing 200-mM sucrose and 2-mM MES ( $\mathrm{pH} 4.2)$ and were later diluted with an outer solution containing 200-mM glucose and 2-mM MES $(\mathrm{pH}$ 4.2). Before confocal microscopy, a fibronectin (FN) solution that had been previously diluted in deionized water was added to make an FN-GUV solution with a final concentration of $10 \mu \mathrm{g} \mathrm{mL}^{-1}$, followed by incubation at $37{ }^{\circ} \mathrm{C}$ overnight. GUVs were imaged by using confocal microscopies (Leica SP8 and ZEISS LSM 710) at the Bioimaging Center of the Advanced Bio-Interface Core Research Facility, Sogang University.

Preparation of Fibronectin (FN): Fibronectin was in powder form before being diluted into deionized water to make a $\mathrm{FN}$ stock solution at concentration of $1 \mathrm{mg} \mathrm{mL}^{-1}$. $\mathrm{FN}$ solution was stored at $-20^{\circ} \mathrm{C}$ or longterm storage and thawed slowly at $4{ }^{\circ} \mathrm{C}$ before use.

FN Conjugation for Fluorescence Resonance Energy Transfer (FRET) Analysis: Fibronectin (FN) was subsequently labeled with Alexa-546 C5 maleimide on its cysteine residues and with Alexa-488 NHS succinimidyl ester on its lysine residues. Lyophilized FN diluted with PBS to a concentration of $1 \mathrm{mg} \mathrm{mL}^{-1}$ was denatured in the chaotropic agent guanidine hydrochloride $(\mathrm{GdnHCl}, 8 \mathrm{M})$ at a ratio of 1:1 $(\mathrm{v} / \mathrm{v})$ and then incubated with Alexa 546 maleimide at a molar ratio of 1:30. Excess fluorescence dye was removed through dialysis by using a slide-A-lyzer dialysis cassette with a 10000 Dalton MW cut-off in 0.1-M sodium bicarbonate $\left(\mathrm{NaHCO}_{3}\right)$ at $\mathrm{pH}$ 8.5. The buffer was changed twice every $2 \mathrm{~h}$ before being stored overnight at $4{ }^{\circ} \mathrm{C}$. The initial conformation of labeled $\mathrm{FN}$ was confirmed using spectrofluorometry. Afterwards, the labeled FN was incubated for one hour with Alexa $488 \mathrm{NHS}$ succinimidyl ester at a ratio of 1:70 ( $\mathrm{mol} / \mathrm{mol})$. Doubly labeled FN with a donor and an acceptor dye (FN-DA) was separated from the free dyes by using a PD-10 column (pre-equilibrated with PBS). The ratio of the donor intensity to the acceptor intensity per $\mathrm{FN}$ dimer $\left(I_{A} / I_{D}\right)$ was determined by measuring the fluorescences of FN-DA at 280,496, and $556 \mathrm{~nm}$. For long-term storage, FN-DA was stored in $10 \%$ glycerol at $-80^{\circ} \mathrm{C}$.

Characterization of FN-SUV Incorporation by Using Cryogenic Transmission Electron Microscopy (Cryo-TEM): FN was incubated overnight with preformed SUVs at a ratio of 1:5 $(w / w)$. Immediately after incubation, samples of either SUVs or FN-SUVs were preserved by vitrification and supported by Quantifoil (R 2/2, 400-mesh holey-carbon grid, Quantifoil Micro Tools). Vitrified samples were prepared by applying a 3- $\mu \mathrm{L}$ drop of sample suspension to a cleaned grid, blotting away the excess with filter paper, and immediately plunging the grid into liquid ethane. Grids were stored under liquid nitrogen until being transferred to the transmission electron microscope for imaging. Electron microscopy was performed using a Talos L120C electron microscope (FEI, Hillsboro, OR, USA) operating at $120 \mathrm{kV}$. Vitreous ice grids were transferred into the electron microscope by using a cryostage that maintained the grids at a temperature below $-170^{\circ} \mathrm{C}$. An image of each grid was acquired on multiple scales to assess the overall distribution of the specimen. Images were collected using a $4 \mathrm{k} \times 4 \mathrm{k}$ Ceta CMOS camera at a search magnification of 5000 and a final magnification of 29000 to 280000 at an underfocus of $\approx 2.5 \mu \mathrm{m}$. After the identification of potentially suitable target areas for imaging at lower magnifications, pairs of highly magnified images were acquired using the UI V1.6 Talos software. Image) software was used to analyze the intralamellar distances in the original, high-resolution images collected using the microscope (4096 $\times 4096$ pixels).

Fluorescence Resonance Energy Transfer (FRET) Analysis: The donor fluorophores Alexa NHS-488 labelled lysine residues and acceptor fluorophores (Alexa maleimide-546) labelled cysteines on FN modules on
$\mathrm{FN}$. There are five lysine and two cysteine residues in each FN monomer. For every FN-FRET batch, we calculated the ratio of donors to acceptors per FN dimer by measuring the absorbances of FN-DA at 280,496, and $556 \mathrm{~nm}$ and using published extinction coefficients for dyes. ${ }^{[57-59]}$ For each dimer molecule used in this study, we calculated an average labeling ratio of $8.5 \pm 0.5$ donors and $3.3 \pm 0.84$ acceptors per FN dimer. Two acceptor-cysteines are presented in FNIII7 and FNIII 15 on each monomer (for molecules for dimer). In native FN (folded), FNIII module locates in cryptic domain stabilizing through ionic interaction between arms; while, in extended FN, FNIII module loss of secondary structure leading distance increase of donor and acceptor $(>12 \mathrm{~nm})$ and decrease the energy transfer.

The fluorescence intensity of FN-DA was measured using a standard fluorescence spectrophotometer (Hitachi F-7000) with excitation of the donor at $488 \mathrm{~nm}$. The positive controls were measured using a series of $\mathrm{GdnHCl}$ concentrations from 0 to $4 \mathrm{M}$. The data from FRET measurements over a range of denaturant concentrations was used to plot the correlation of the FRET efficiency with the degree of FN unfolding. [60] FN-DA was incubated overnight with preformed SUVs at a ratio of 1:5 $(\mathrm{w} / \mathrm{w})$ with gentle rocking to enhance the binding. Then, cryo-TEM and FRET were exploited to characterize the incorporation and the unfolding of FN on the SUVs. The background fluorescence was measured using SUVs in solution, and the negative control was measured using FN-DA in PBS at the same concentration as in the FN-SUV sample, but without SUVs.

Colocalization, Attachment Area, and Fibril Formation Imaging: HDFn cells were cultured in DMEM (FBS 10\%, 1\% Pen-Strep). For integrin observation, FITC-conjugated integrin $\alpha 5$ antibody was used. FN was diluted in PBS and used at a concentration of $10 \mu \mathrm{g} \mathrm{mL} \mathrm{L}^{-1}$. For cell attachment experiments, HDFn-GFP cells were detached using trypsin-EDTA when the confluence had reached $80 \%$ and were then transferred to a new culture dish, treated with non-labeled FN and imaged every hour in an on-stage incubator. For quantification of the area of the FN fibrils, HDFn-GFP cells were treated with lissamine-rhodamine-labeled FN and imaged. In-situ images as a function of time were taken using an on-stage incubator with $\mathrm{CO}_{2}$ and temperature control. Images and videos were processed using Image) and Zen software.

In Vitro Scratch Assay: In migration experiments, GFP-HDFn cells were cultured in T75 flasks to reach a confluence of $80-90 \%$ and were then transferred to $35-\mathrm{mm}$ dishes for continuous culturing over $24 \mathrm{~h}$ with $10 \%$ FBS for attachment. When cells had formed a confluent monolayer, the medium was replaced with low-serum DMEM (0.4\% FBS) every $24 \mathrm{~h}$ to minimize the effects of proliferation on the migration speed and to avoid unexpected apoptosis. ${ }^{[41]}$ The distance that a single cell moved was tracked using ImageJ.

A scratch mimicking a wound was created using a p200 pipette tip by scraping a straight line across the center of the dish. Cell debris was then washed away gently with $2 \mathrm{~mL}$ of low-serum medium before being supplemented with either PBS, FN, or FN-SUV. The final concentration of FN or FN-SUV solution is $10 \mu \mathrm{g} \mathrm{mL}^{-1}$ in all experiments, unless otherwise stated. The $35-\mathrm{mm}$ dish was placed in a stage-top incubator (Zeiss, USA) with a $\mathrm{CO}_{2}$ supply and a controlled temperature of $37^{\circ} \mathrm{C}$ to obtain the same optical field during the image acquisition. Images of GFP-HDFn cells migrating to the scratch were recorded using confocal microscopy (ZEISS LSM 710, USA) in a time series mode at $1 \mathrm{~h}$ intervals for at least $15 \mathrm{~h}$.

Ulcerative Colitis Rat Model: We followed the Guidelines for the Care and Use of Animals approved by the National Institute of Health Council for the management and use of animals for laboratory tests. The experimental design was approved by the Institutional Care and Use Committee of Kyung Hee University (KHUASP(SE)-15-085). Rats were divided into the following five groups ( $n=10$ in each group): healthy group (negative control), ulcerative colitis (UC)-induced group (positive control), UCinduced/mesalamine-treated group, UC-induced/FN-SUV-treated group, and UC-induced/FN-treated group.

Induction of Ulcerative Colitis by Using Acetic Acid: Ulcerative colitis (UC) was induced by using acetic acid, as previously described. [61] The rats were anesthetized with Zoletil 50 ( $10 \mathrm{mg} \mathrm{kg}^{-1}$; Vibac Laboratories, Carros, 
France). Animals were fasted for $48 \mathrm{~h}$ for UC preparation. A stainless-steel feeding needle (round tip, 16 gauge) was then carefully inserted into the colon of a rat with the tip being $8 \mathrm{~cm}$ proximal to the anus. Then, $4 \%$ acetic acid (Sigma Chemical Co., St. Louis, MO, USA) in $0.9 \%$ saline $(1.0 \mathrm{~mL})$ was injected into the lumen of the colon. The control group underwent intracolonic injection with saline instead. The rats were maintained in a supine Trendelenburg position for $5 \mathrm{~min}$ to prevent leakage of the injected acetic acid solution.

UC Treatment: The UC treatments were administered three days after acetic acid injection. The dosages for the groups were as follows: mesalamine $(4 \mathrm{~g} / 100 \mathrm{~mL}$, Asacol Enema, Tillotts Pharma, AG, Swiss), FN-

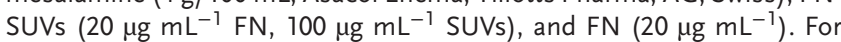
drug administration, the rats were confined in an acrylic cylinder (diameter: $6 \mathrm{~cm}$, length: $20 \mathrm{~cm}$, height: $10 \mathrm{~cm}$ ) without anesthesia. A stainlesssteel feeding needle (round tip, 16 gauge) with a syringe was then carefully inserted through the anus into the damaged site. Then, each drug $(1.0 \mathrm{ml})$ was injected into the lumen of the colon once a day for 10 days after induction of UC.

HQE Staining and Evaluated of Ulcer Score: Histological changes in colon tissues were detected by $\mathrm{H} \& \mathrm{E}$ staining as previously described by Jeon et al. ${ }^{[62]}$ The slides were immersed in Mayer's hematoxylin (DAKO, Glostrup, Denmark) for $30 \mathrm{~s}$ and then rinsed with tap water until clean. The slides were then immersed in eosin (Sigma Chemical Co., St. Louis, MO, USA) for $10 \mathrm{~s}$ and rinsed again with water. The slides were dried at room temperature and then immersed in $95 \%$ ethanol twice, in $100 \%$ ethanol twice, in $50 \%$ ethanol and $50 \%$ xylene twice, and in $100 \%$ xylene twice. The slides were covered with coverslips of Permount (Fisher Scientific, New Jersey, NJ, USA). The parameters evaluated for ulcer score were: histological ulceration, mucosal atrophy, submucosal edema, and inflammatory cells infiltration. Each parameter was scored on a scale from zero (normal) to 3 (severe damage) according to the criteria described by Mehrabani et al. .[63] Total ulcer score was calculated by summing up individual scores for each rat. ${ }^{63,64]}$

Western Blotting: Colon tissues were homogenized on ice, lysed with lysis buffer, and then centrifuged at $14000 \mathrm{rpm}$ for $30 \mathrm{~min}$. The amount of protein in each sample was quantified using a Bio-Rad colorimetric protein assay kit (Bio-Rad, Hercules, CA, USA). Sodium dodecyl sulfatepolyacrylamide gels were used to separate proteins $(30 \mu \mathrm{g})$, after which the separated proteins were transferred onto nitrocellulose membranes. Goat COX-2 antibody (1:1000; Santa Cruz Biotechnology, Santa Cruz, CA, USA), mouse iNOS antibody, and mouse $\beta$-actin antibody (1:1000; Santa Cruz Biotechnology) were used as the primary antibodies. Horseradish peroxidase-conjugated anti-mouse antibodies (1:2000; Vector Laboratories, Burlingame, CA, USA) for iNOS, $\beta$-actin, and COX- 2 were used as secondary antibodies. Membrane transfer was conducted at $4{ }^{\circ} \mathrm{C}$ by using a cold pack and pre-chilled buffer. An enhanced chemiluminescence (ECL) detection kit (Santa Cruz Biotechnology) was used for band detection. Molecular Analyst (version 1.4.1, Bio-Rad) was used to analyze the detected bands.

Data Analysis: All of the images, except for the in vivo experiments, were analyzed using either Image) (1.51) or Zen software (2.3 SP1, Black edition). Migration of each cell was analyzed by using the plug-in Mtrack2 in Image). The recovery areas were quantified using Image). For cell area study, the intensity of GFP-expressing HDFn per area was calculated from the confocal images by using Image). The orientation in degrees of greyscale GFP-expressing HDFn images were analyzed through the Orientationj plug-in Imagel software.

Using the Image-Pro plus computer-assisted image analysis system (Media Cybernetics Inc., Silver Spring, MD, USA), the area of UC in each sample was measured with help of a light microscope (Olympus, Tokyo, Japan). For relative quantification, the results in the control group of western blot were set as 1.00 .

\section{Supporting Information}

Supporting Information is available from the Wiley Online Library or from the author.

\section{Acknowledgements}

K.Y.L and H.T.N. contributed equally. This work was supported by the Samsung Science and Technology Foundation under Project Number SSTFBA 800112010 . International collaboration was in part conducted through the Sogang-Harvard-SDU Biohybrid Center.

\section{Conflict of Interest}

The authors declare no conflict of interest.

\section{Data Availability Statement}

Research data are not shared.

\section{Keywords}

extracellular matrix, fibronectin, fibronectin delivery, small unilamellar vesicles, ulcerative colitis, wound healing

Received: August 4, 202

Revised: October 26, 2021

Published online:

[1] W. S. To, K. S. Midwood, Fibrog. Tissue Repair 2011, 4, 21.

[2] M. Gao, D. Craig, V. Vogel, K. Schulten, J. Mol. Biol. 2002, 323, 939.

[3] C. A. Lemmon, S. H. Weinberg, Sci. Rep. 2017, 7, 18061.

[4] E. P. Gee, D. Yuksel, C. M. Stultz, D. E. Ingber, J. Biol. Chem. 2013, $288,21329$.

[5] L. M. Maurer, W. Ma, D. F. Mosher, Crit. Rev. Biochem. Mol. Biol. 2015, 51,213

[6] J. A. Paten, C. L. Martin, J. T. Wanis, S. M. Siadat, A. M. FigueroaNavedo, J. W. Ruberti, L. F. Deravi, Chem 2019, 5, 1930.

[7] J. E. Schwarzbauer, J. L. Sechler, Curr. Opin. Cell Biol. 1999, 11, 622.

[8] J. Ulmer, B. Geiger, J. P. Spatz, Soft Matter 2008, 4, 1998.

[9] H. W. Verspaget, I. Biemond, C. F. Allaart, H. van Weede, I. T. Weterman, H. G. Gooszen, A. S. Pena, C. B. Lamers, Hepatogastroenterology 1991, 38, 231.

[10] M. L. Smith, D. Gourdon, W. C. Little, K. E. Kubow, R. A. Eguiluz, S Luna-Morris, V. Vogel, PLoS Biol. 2007, 5, e268.

[11] K. J. Johnson, H. Sage, G. Briscoe, H. P. Erickson, J. Biol. Chem. 1999, 274, 15473

[12] V. Vogel, Annu. Rev. Biophys. Biomol. Struct. 2006, 35, 459.

[13] O. S. Ejim, G. W. Blunn, R. A. Brown, Biomaterials 1993, 14, 743.

[14] J. Patten, K. Wang, Adv. Drug Delivery Rev. 2021, 170, 353.

[15] Z. Qiu, A. H. Kwon, Y. Kamiyama, J. Surg. Res. 2007, 138, 64.

[16] M. B. Johnson, B. Pang, D. J. Gardner, S. Niknam-Benia, V. Soundarajan, A. Bramos, D. P. Perrault, K. Banks, G. K. Lee, R. Y. Baker, G. H. Kim, S. Lee, Y. Chai, M. Chen, W. Li, L. Kwong, Y. K. Hong, A. K. Wong, Sci. Rep. 2017, 7, 3876

[17] M. Nakamura, T. Nishida, M. Hikida, T. Otori, Curr. Eye Res. 1994, 13 , 385.

[18] S. Ahn, L. F. Deravi, S. J. Park, B. E. Dabiri, J. S. Kim, K. K. Parker, K. Shin, Adv. Mater. 2015, 27, 2838.

[19] S. Jordahl, L. Solorio, D. B. Neale, S. McDermott, J. H. Jordahl, A. Fox, C. Dunlay, A. Xiao, M. Brown, M. Wicha, G. D. Luker, J. Lahann, Adv. Mater. 2019, 31, 1904580.

[20] N. Pernodet, M. Rafailovich, J. Sokolov, D. Xu, N. L. Yang, K. McLeod, J. Biomed. Mater. Res., Part A 2003, 64, 684.

[21] P. Le, S.-N. Mai-Thu, T.-H. Nguyen, T. Vo Van, K. Huynh, presented at IFMBE Proc., Ho Chi Minh, Vietnam, May 2017. 
[22] C. O. Chantre, P. H. Campbell, H. M. Golecki, A. T. Buganza, A. K. Capulli, L. F. Deravi, S. Dauth, S. P. Sheehy, J. A. Paten, K. Gledhill, Y. S. Doucet, H. E. Abaci, S. Ahn, B. D. Pope, J. W. Ruberti, S. P. Hoerstrup, A. M. Christiano, K. K. Parker, Biomaterials 2018, 166, 96.

[23] D. B. Neale, A. J. Muñiz, M. S. Jones, D. H. Kim, J. M. Buschhaus, B. A. Humphries, W. Y. Wang, B. M. Baker, J. E. Raymond, L. Solorio, G. D. Luker, J. Lahann, Small Struct. 2021, 2, 2000137.

[24] R. J. Klebe, K. L. Bentley, R. C. Schoen, J. Cell. Physiol. 1981, 109, 481.

[25] M. Lin, H. Wang, C. Ruan, J. Xing, J. Wang, Y. Li, Y. Wang, Y. Luo Biomacromolecules 2015, 16, 973.

[26] G. K. Toworfe, R. J. Composto, C. S. Adams, I. M. Shapiro, P. Ducheyne, J. Biomed. Mater. Res., Part A 2004, 71, 449.

[27] A. L. Koenig, V. Gambillara, D. W. Grainger, J. Biomed. Mater. Res. Part A 2003, 64, 20.

[28] A. W. Feinberg, K. K. Parker, Nano Lett. 2010, 10, 2184.

[29] S. Patel, A. F. Chaffotte, F. Goubard, E. Pauthe, Biochemistry 2004, 43, 1724.

[30] J. A. Weston, K. L. Hendricks, Proc. Natl. Acad. Sci. U. S. A. 1972, 69, 3727.

[31] A. D. Glinos, G. N. Bardi, K. C. Dermitzaki, S. A. Perez, M. J. Talieri, J. Natl. Cancer Inst. 1983, 71, 1211.

[32] R. Mezzenga, M. Mitsi, Biomacromolecules 2019, 20, 55.

[33] O. D. Monera, C. M. Kay, R. S. Hodges, Protein Sci. 1994, 3, 1984.

[34] A. J. Garclá, M. a. D. Vega, D. Boettiger, Mol. Biol. Cell 1999, 10, 785.

[35] B. Lariviere, M. Rouleau, S. Picard, A. D. Beaulieu, Wound Repair Regener. 2003, 11, 79.

[36] Y. X. Liao, Z. P. Zhang, J. Zhao, J. P. Liu, Cell. Physiol. Biochem. 2018, 48, 1382.

[37] A. R. Toledo-Piza, E. Nakano, R. E. Rici, D. A. Maria, Cell Proliferation 2013, 46, 97.

[38] M. Chabria, S. Hertig, M. L. Smith, V. Vogel, Nat. Commun. 2010, 1 135.

[39] L. E. Tracy, R. A. Minasian, E. J. Caterson, Adv. Wound Care 2016, 5, 119.

[40] R. A. Clark, J. M. Lanigan, P. DellaPelle, E. Manseau, H. F. Dvorak, R. B. Colvin, J. Invest. Dermatol. 1982, 79, 264.

[41] C. C. Liang, A. Y. Park, J. L. Guan, Nat. Protoc. 2007, 2, 329.

[42] A. H. Kwon, Z. Qiu, Y. Hiraon, Surgery 2007, 141, 254.
[43] F. Grinnell, R. E. Billingham, L. Burgess, J. Invest. Dermatol. 1981, 76, 181.

[44] R. Kochhar, N. Kumar, V. Singh, M. K. Goenka, S. K. Mehta, Natl. Med. J. India 1991, 4, 108

[45] J.-d. Luo, A. F. Chen, Acta Pharmacol. Sin. 2005, 26, 259

[46] A. Futagami, M. Ishizaki, Y. Fukuda, S. Kawana, N. Yamanaka, Lab. Invest. 2002, 82, 1503.

[47] G. Guo, W. Shi, F. Shi, W. Gong, F. Li, G. Zhou, J. She, J. Biochem. Mol. Toxicol. 2019, 33, e22400.

[48] J. M. Szymanski, E. N. Sevcik, K. Zhang, A. W. Feinberg, Biomater. Sci. 2017, 5, 1629.

[49] V. Vadillo-Rodriguez, J. M. Bruque, A. M. Gallardo-Moreno, M. L. Gonzalez-Martin, Langmuir 2013, 29, 8554.

[50] M. Bergkvist, J. Carlsson, S. Oscarsson, J. Biomed. Mater. Res., Part A 2003, 64, 349.

[51] M. R. Mozafari, C. J. Reed, C. Rostron, Pharmazie 2007, 62, 205.

[52] A. M. Reichmuth, M. A. Oberli, A. Jaklenec, R. Langer, D. Blankschtein, Ther. Delivery 2016, 7, 319.

[53] S. Hossen, M. K. Hossain, M. K. Basher, M. N. H. Mia, M. T. Rahman, M. J. Uddin, J. Adv. Res. 2019, 15, 1

[54] U. Bulbake, S. Doppalapudi, N. Kommineni, W. Khan, Pharmaceutics 2017, 9, 12.

[55] G. Smistad, J. Jacobsen, S. A. Sande, Int. J. Pharm. 2007, 330, 14.

[56] M. Halter, M. Antia, V. Vogel, J. Controlled Release 2005, 101, 209.

[57] D. Ortiz Franyuti, M. Mitsi, V. Vogel, Nano Lett. 2018, 18, 15.

[58] H. P. Erickson, Proc. Natl. Acad. Sci. U. S. A. 1994, 91, 10114.

[59] E. M. Chandler, M. P. Saunders, C. J. Yoon, D. Gourdon, C. Fischbach, Phys. Biol. 2011, 8, 015008.

[60] G. Baneyx, L. Baugh, V. Vogel, Proc. Natl. Acad. Sci. USA 2002, 99, 5139.

[61] A. M. Schoepfer, C. Beglinger, A. Straumann, M. Trummler, P. Renzulli, F. Seibold, Inflammatory Bowel Dis. 2009, 15, 1851.

[62] J. W. Jeon, J. I. Lee, H. P. Shin, J. M. Cha, K. R. Joo, S.-H. Kim, I.-G. Ko, J.-J. Jin, S.-E. Kim, C.-J. Kim, Animal Cells Syst. 2014, 18, 399.

[63] D. Mehrabani, M. Ziaei, S. V. Hosseini, L. Ghahramani, A. M. Bananzadeh, M. J. Ashraf, A. Amini, M. Amini, N. Tanideh, Iran Red Crescent Med. J. 2011, 13, 884.

[64] H. M. El-Gowelli, E. I. Saad, A. G. Abdel-Galil, E. R. Ibrahim, Toxicol. Appl. Pharmacol. 2015, 288, 300. 